04

\title{
Сравнение вариантов литиевого дивертора для токамака ДЕМО-ТИН
}

\author{
(C В.Г. Скоков, ${ }^{1}$ В.Ю. Сергеев, ${ }^{1}$ Е.А. Ануфрриев, ${ }^{1}$ Б.В. Кутеев ${ }^{2,3}$ \\ ${ }^{1}$ Санкт-Петербургский политехнический университет Петра Великого, \\ 195251 Санкт-Петербург, Россия \\ ${ }^{2}$ Национальный исследовательский центр „Курчатовский Институт“, \\ 123182 Москва, Россия \\ ${ }^{3}$ Национальный исследовательский ядерный университет „МИФИ“, \\ 115409 Москва, Россия \\ e-mail: V.Skokov@spbstu.ru
}

Поступило в Редакцию 03 сентября 2020 г.

В окончательной редакции 18 ноября 2020 г.

Принято к публикации 19 ноября 2020 г.

Для разрабатываемого в России токамака ДЕМО-ТИН обсуждается выбор концепции дивертора с испаряющимся жидким литием, удовлетворяющей требованиям по снятию тепловой нагрузки от пристеночной плазмы и обеспечивающей приемлемый уровень изменения ионного состава основной плазмы. Представлены результаты численного моделирования и оптимизации конструкции диверторов с несколькими объемами, секционированными щелевыми диафрагмами. Оценены параметры литиевых потоков, вытекающих в пристеночный слой, для интервала изменения температуры диверторных камер от 500 до $1000 \mathrm{~K}$ в условиях газокинетических и свободномолекулярных режимов истечения литиевого пара из дивертора. Проведенный анализ процессов, снижающих выход лития из камер и проникновение его в основной объем плазмы внутри сепаратрисы, показал, что секционирование эффективно снижает вытекающие потоки до приемлемых уровней $\sim 10^{20}$ at $/ \mathrm{s}$.

Ключевые слова: дивертор, литий, тепловая нагрузка, ДЕМО-ТИН.

DOI: $10.21883 /$ JTF.2021.04.50619.259-20

\section{Введение}

Вопросы отвода тепла, приносимого плазмой в дивертор токамака, продолжают оставаться нерешенной инженерно-физической проблемой создания термоядерного энергетического реактора [1] и термоядерного источника нейтронов [2], в том числе компактного [3].

В экспериментах на современных токамаках обнаружено, что поперечный размер диверторного слоя Scrape-Off Layer (SOL) в области взаимодействия с элементами дивертора слабо зависит от размеров установки и уменьшается с ростом тока плазмы [4]. Например, для ИТЭР с током 15MA размер SOL в экваториальной плоскости со стороны слабого магнитного поля (upstream) оценивается как $1 \mathrm{~mm}$ [4]. В результате для демонстрационного термоядерного реактора продольные потоки тепла $\approx 20 \mathrm{GW} / \mathrm{m}^{2}[5]$ при выходе на материальную поверхность дают значения нормального к ней теплового потока, которые на порядки превышают приемлемые инженерные значения $\approx 10 \mathrm{MW} / \mathrm{m}^{2}[1]$.

Для снижения теплового потока на диверторные пластины было предложено много подходов [6,7]. Напуск инертных газов для достижения режима полного детачмента (отрыва плазмы от поверхности) позволяет переизлучить значительную часть мощности, но сопровождается охлаждением области $X$-точки и, как следствие, центральной области плазмы. Для создания так называемых диверторов с длинной ногой сепаратрисы
(super-X divertor) [8] и других сложных магнитных диверторных конфигураций типа снежинки (snow-flake) [9] необходимо располагать дополнительные катушки внутри вакуумной камеры и защищать их от нейтронных потоков. В результате конструкция установки и технологии обслуживания дивертора заметно усложняются.

Был выдвинут ряд предложений [10-12] по использованию систем с жидкими металлами (литием, оловом) для защиты обращенных к плазме поверхностей от эрозии, организации теплоотвода в периферийной плазме и управления составом примесей. При этом жидкий металл может непосредственно взаимодействовать с периферийной плазмой разряда. Одной из модификаций являются лимитеры с капиллярно-пористыми структурами (КПС) (см., например, [13]), в которых жидкий металл удерживается на поверхности капиллярными силами. На установке NSTX [14] литиевая жидкометаллическая пластина испытывалась в открытой диверторной конфигурации. Литий был жидкий и находился в ваннах, формировал нижний однонулевой дивертор за счет силы тяжести. Следует заметить, что длина ноги в однонулевом диверторе установки была небольшой, и жидкий литий находится вблизи сепаратрисы. Ряд конструкций с жидким литием на поверхности испытывался на установках без диверторов в лимитерной конфигурации плазмы. Таким образом, практически во всех экспериментах периферийная плазма взаимодействовала с открытыми литиевыми жидкометаллическими поверхностями, с ко- 
торых трудно ограничить поток лития в плазму при росте тепловой нагрузки.

В условиях высоких плазменных нагрузок, характерных для будущих термоядерных установок, такие варианты выглядят неадекватными. Наиболее перспективными на сегодня представляются подходы, основанные на испарении жидкого лития потоками тепла из пристеночной плазмы и со стенок в замкнутом объеме, предложенные Нагаямой [15] и Голдстоном [5]. Они относительно просты в реализации и представляются способными достичь необходимого стационарного режима для потоков тепла и частиц плазмы из основного объема в диверторную область.

Нагаяма в своей работе [15] предложил разместить в диверторе токамака-реактора две последовательные тороидально-симметричные камеры, связанные с плазмой и друг с другом щелевыми отверстиями, через которые плазма SOL доходит до диверторных пластин с поверхностью в виде жидкого лития. Температура стенок двухкамерной конструкции поддерживается внешними системами охлаждения выше уровня температуры плавления лития $\left(\approx 180^{\circ} \mathrm{C}\right)$, обеспечивая жидкое состояние лития. Основная мощность SOL-плазмы, втекающая в камеры дивертора, снимается за счет испарения лития в области точки удара (strike point). В первой камере предполагается поглощение основного потока паров стенками. Часть образующихся литиевых паров должна осаждаться на холодных стенках, другая часть пролетает через щель между камерами испарителя. В работе предполагается, что давление пара в камере будет при указанных температурах достаточно низким для реализации условий свободно-молекулярного разлета в заметной части объема и области выходной щели. В таких условиях число выходящих в SOL литиевых частиц определяется апертурой выходного отверстия, a также плотностью и температурой атомов лития. Предусмотрена также система откачки, очистки загрязненного лития и пополнения бассейна. Этот вариант дивертора ниже будет называться подходом с конденсирующими литий стенками.

Голдстон, в свою очередь, предложил похожую конструкцию дивертора для токамака-реактора ДЕМО [5], состоящую из 5 последовательных тороидальносимметричных камер, которые также соединены между собой щелями, через которые проходит сепаратриса. Каждая из камер наполнена парами лития при определенных температурах стенок и соответствующих давлениях. Эти пары способны переизлучить заметную часть мощности, приходящей в дивертор из SOL. Paдиационные потери плазмы на литиевых парах Голдстон оценивал, используя среднюю энергию излучения на один атом лития $\approx 10 \mathrm{eV}$, исходя из расчетов столкновительно-излучательной модели [16].

Последовательность камер представляет собой дифференциальную систему откачки: давление литиевых паров падает примерно на порядок при переходе от камеры к камере при движении в сторону основной плазмы.
Температуры стенок камер для создания плотных литиевых паров поддерживаются при достаточно высоких значениях (до $950^{\circ} \mathrm{C}$ ). В связи с этим ниже данный вариант будет именоваться подходом с испаряющими стенками.

В настоящей работе проводится сравнительный анализ и оцениваются возможности применения обоих подходов для дивертора, разрабатываемого в проекте Демонстрационного термоядерного источника нейтронов (ДЕМО-ТИН) [17,18]. В разд. 1 приведены параметры проекта ДЕМО-ТИН и описаны ключевые критерии выбора параметров дивертора. Конструкции диверторной зоны, а также оценки их параметров в подходе испаряющих и конденсирующих стенок диверторных камер приведены в разд. 2 и 3 соответственно. В разд. 4 обсуждаются достижимые параметры диверторов в рамках двух подходов. В заключении суммированы основные результаты работы.

\section{1. Установка ДЕМО-ТИН}

Разрабатываемый в настоящее время сверхпроводящий токамак ДЕМО-ТИН [17,18] имеет следующие основные параметры: большой радиус $R=320 \mathrm{~cm}$, малый радиус $a=100 \mathrm{~cm}$, треугольность $\delta=0.41$, ток по плазме $I_{p}=4.5 \mathrm{MA}$, тороидальное магнитное поле $B_{t}=5 \mathrm{~T}$, электронная и ионная температуры в центре $T_{e, i}(0) \cong 11 \mathrm{keV}$, средняя плотность $\langle n\rangle=8 \cdot 10^{13} \mathrm{~cm}^{-3}$. Мощность DT-синтеза в стационарном режиме должна составлять до $40 \mathrm{MW}$, нейтронный выход - свыше $1 \cdot 10^{19} \mathrm{~s}^{-1}$. Температура плазмы и ее плотность в SOL были оценены как: $T_{p l}=150 \mathrm{eV}, n_{p l}=4 \cdot 10^{13} \mathrm{~cm}^{-3}$. Указанные данные получены в результате описанного в работе [19] гибридного моделирования ДЕМО-ТИН с помощью кодов SOLPS4.3 и ASTRA. Диверторная конфигурация - двухнулевая, ноги дивертора со стороны слабого и сильного магнитного полей принято называть „внешней“ и „внутренней“ соответственно. Тепловая нагрузка в этой конфигурации будет симметрично делиться между верхним и нижним диверторами. Между внешней и внутренней ногами каждого дивертора мощность будет распределяться в отношении 10 и $3 \mathrm{MW}$ [19-21]. При этом тепловой поток вдоль магнитного поля на входе в дивертор будет достигать значений $\sim 1 \mathrm{GW} / \mathrm{m}^{2}$. Очевидно, что необходимо найти решение, приводящее к снижению на несколько порядков потока тепла на материальные поверхности дивертора.

Эскиз трехкамерной конструкции с жидким литием для внешней и внутренней ног нижнего дивертора можно найти в работе [18]. Для обсуждения расчета параметров в обоих подходах на рис. 1 показан фрагмент данной конструкции для внешнего нижнего дивертора. Номера камер дивертора обозначены цифрами $i=1-3$, начиная с дальней от плазмы. В данном варианте длины всех камер $L_{i}$ вдоль сепаратрисы составляют $7.5 \mathrm{~cm}$, а ширины щелей поперек сепаратрисы $H_{i}=5 \mathrm{~cm}$. Для 


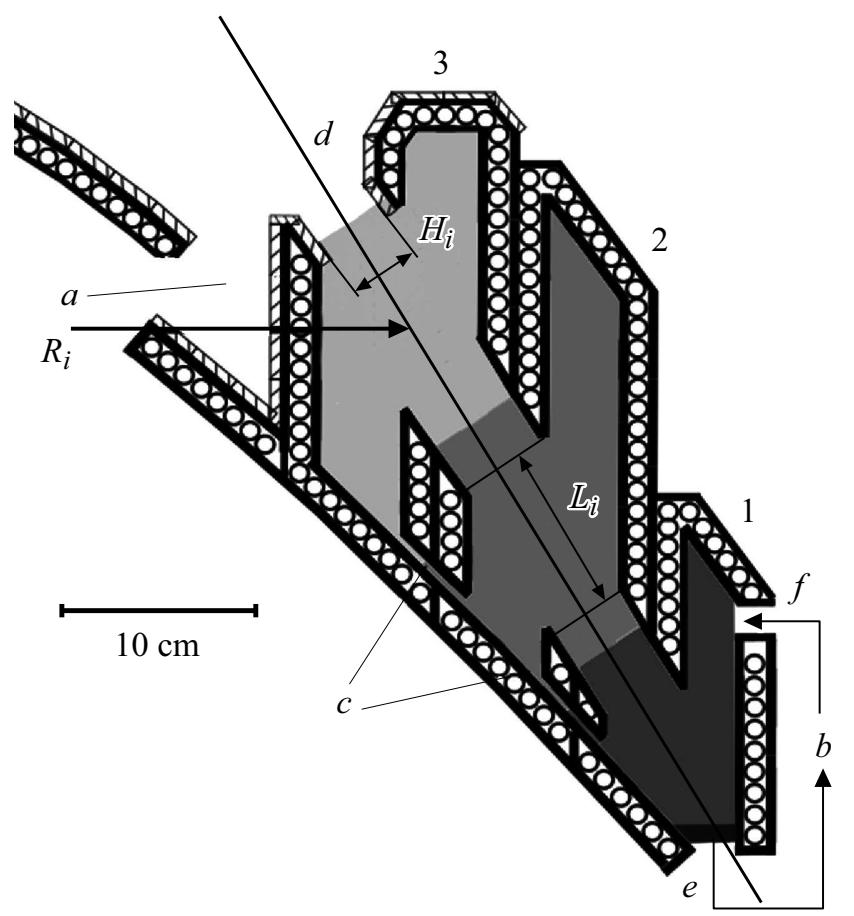

Рис. 1. Схема литиевого дивертора ДЕМО-ТИН. Цифрами показаны номера камер. $a-$ щели для откачки, $b-$ система очистки и перекачки лития, $c$ - щели для стекания жидкого лития в камеру $1, d-$ сепаратриса, $e$ и $f-$ выход лития в систему очистки и перекачки, и вход чистого лития в дивертор соответственно. Насыщенность серого цвета иллюстрирует относительное давление лития в камерах. $R_{i}$ и $L_{i}-$ усредненный большой радиус сепаратрисы в $i$-й камере и длина камеры соответственно, $H_{i}$ - ширина щели.

оценки ширины щели учитывалась конечная ширина SOL, а также возможные колебания сепаратрисы в плоскости, перпендикулярной щели, из-за переходных процессов [2] и несовершенства системы управления положением плазменного шнура.

Обращенная к основной плазме поверхность последней камеры дивертора, равно как и его купол, должны быть покрыты бериллиевыми тайлами (на рис. 1 показаны заштрихованными прямоугольниками; о мотивации выбора материала см. [21]). В диверторе предусматриваются отверстия для откачки (позиция $a$ на рис. 1), а также выход для сконденсировавшегося лития, насыщенного рабочим газом и примесями $e$, в систему очистки и перекачки лития $b$. Очищенный литий подается обратно в дивертор через отверстие $f$. В нижней части камеры 1 расположен бассейн с расплавом Li. Относительные изменения давления паров лития на рисунке схематично показаны плотностью заливки серого цвета.

Для сопоставления диверторных систем в ДЕМО-ТИН для обоих подходов $[5,15]$ было выработано четыре критерия, которые перечислены ниже в порядке убывания их значимости. Первый - результирующие тепловые нагрузки на материальные поверхности не должны превышать $10 \mathrm{MW} / \mathrm{m}^{2}$.
Второй - приемлемый поток лития через сепаратрису внутрь основной плазмы, который не приводит к значительному загрязнению примесью. Для средних значений концентрации рабочего газа $-6.4 \cdot 10^{19} \mathrm{~m}^{-3}$, гелия - $6.4 \cdot 10^{18} \mathrm{~m}^{-3}$ и основной примеси Ве $3.2 \cdot 10^{18} \mathrm{~m}^{-3}$ средняя плотность электронов составляет $8 \cdot 10^{19} \mathrm{~m}^{-3}$, а эффективный заряд $Z_{\mathrm{eff}} \approx 1.6$. Добавление лития до значений $6.4 \cdot 10^{18} \mathrm{~m}^{-3}(10 \%$ от плотности рабочего газа) незначительно увеличивает эффективный заряд до приемлемого значения 1.8. Время удержания частиц $\approx 2$ s в ДЕМО-ТИН можно оценить, предположив его равным трехкратному времени удержания энергии аналогично работе [19]. Тогда с учетом объема плазмы $120 \mathrm{~m}^{3}$ поток лития через сепаратрису составит: $6.4 \cdot 10^{19} \mathrm{~m}^{-3} \cdot 0.1 \cdot 120 \mathrm{~m}^{3} \cdot(2 \cdot c)^{-1} \cong 3.85 \cdot 10^{20} \mathrm{at} / \mathrm{s}$, т. е. $\cong 4.5 \mathrm{mg} / \mathrm{s}$. Таким образом, поток лития, выходящий из последней камеры дивертора, должен создавать поток в основной объем плазмы не более $4 \cdot 10^{20} \mathrm{at} / \mathrm{s}$.

С другой стороны, необходимо, чтобы выходящий из диверторов поток лития не приводил также и к значительному охлаждению плазмы SOL. Ионизационные потенциалы нейтралов и ионов лития составляют 5.39, 75.6 и $123 \mathrm{eV}$ соответственно. В работе [16] излучательные потери в плазме с температурой $150 \mathrm{eV}$ составляют порядка $100 \mathrm{eV}$ на один атом лития. C учетом необходимости нагрева образовавшихся электронов и иона до $T_{\mathrm{SOL}}=150 \mathrm{eV}$ потери на ионизацию, излучение и термализацию атома Li, попадающего из дивертора в SOL, можно оценить как $100+5.39+75.6+123+4 \cdot 150 \approx$ $\approx 904 \mathrm{eV}$. Выходящая из основной плазмы в SOL суммарная мощность составляет $2 \cdot(3+10)=26 \mathrm{MW}$. Предположив, что на термализацию лития тратится не более $10 \%$ от этой мощности, можно оценить допустимый поток лития из диверторов в SOL $2.0 \cdot 10^{22} \mathrm{at} / \mathrm{s}=$ $=2.6 \mathrm{MW} / 904 \mathrm{eV}$ или $0.21 \mathrm{~g} / \mathrm{s}$ лития.

Третий критерий - предлагаемый литиевый дивертор не должен выходить за габариты современного проекта вакуумной камеры ДЕМО-ТИН. Четвертый критерий возможность инженерно-технической реализации каждой из систем с использованием доступных материалов и технологий. В следующих двух разделах представлены результаты расчетов и оценки для двух вариантов диверторной системы ДЕМО-ТИН, с помощью которых на основании перечисленных критериев будет осуществляться выбор оптимального подхода.

\section{2. Оценки для дивертора ДЕМО-ТИН в подходе с испаряющими стенками}

Предлагаемая диверторная система рассчитывалась с использованием модели, в которую входили стационарные нульмерные уравнения баланса атомов лития и энтальпии литиевого пара для каждой камеры [5]. Были рассчитаны параметры модели с испаряющими стенками в вариантах с количеством камер от 2 до 4. 
В [16] было показано, что в рабочем режиме разница давлений между камерами будет достаточной для формирования критического потока между ними. Согласно [22], можно записать выражение для критического потока паров лития из $i$-й камеры:

$$
\Gamma_{n, i}=\xi_{\mathrm{Li}} n_{i} \sqrt{k T_{\text {vap }, i} / m_{\mathrm{Li}}}
$$

где $k$ - постоянная Больцмана, $T_{v a p, i}$ - температура газа вдали от щели, $m_{\mathrm{Li}}$ - масса атома лития, $n_{i}-$ плотность газа, $\xi_{\mathrm{Li}}=\sqrt{\gamma} \cdot\left(\frac{2}{\gamma+1}\right)^{(\gamma+1) /(2(\gamma 1))}=0.7262$, где $\gamma=5 / 3$ показатель адиабаты для паров лития (см. [5,23]).

На стенки камеры падает ленгмюровский поток

$$
\Gamma_{l, i}=n_{i} \sqrt{k T_{\text {vap }, i} / 2 \pi m_{\mathrm{Li}}},
$$

а со стенок испаряется поток

$$
\Gamma_{a, i}=n_{e q} \sqrt{k T_{w a l l, i} / 2 \pi m_{\mathrm{Li}}}=p / \sqrt{2 \pi m_{\mathrm{Li}} k T_{w a l l, i}} .
$$

Здесь $T_{\text {wall,i }}$ - температура стенки $i$-й камеры. Поток определяется давлением насыщенного пара лития в камере с испаряющими стенками, который зависит от температуры [24]:

$$
\lg p=10.015-\frac{8064.6}{T_{\text {wall }, i}},
$$

где давление выражается в паскалях, температура в градусах Кельвина.

В работе [16] были представлены результаты моделирования 5-камерного литиевого дивертора с помощью уравнений Навье-Стокса. Они показали, что задачу можно рассматривать приближенно, предполагая температуры и плотности паров лития однородными в каждой из камер, а также считая, что частицы пролетают сквозь щели из более горячей камеры в более холодную со звуковой скоростью. Тогда с помощью (1)-(3) уравнение баланса частиц (4) может быть записано для $i$-й камеры следующим образом:

$$
\begin{aligned}
& \xi_{\mathrm{Li}} \sqrt{\frac{k}{m_{\mathrm{Li}}}}\left(A_{n o z, i-1} n_{i-1} \sqrt{T_{\text {vap }, i-1}}-A_{n o z, i} n_{i} \sqrt{T_{\text {vap }, i}}\right) \\
& +\sqrt{\frac{k}{2 \pi m_{\mathrm{Li}}}} A_{\text {wall }, i}\left(n_{e q}\left(T_{\text {wall }, i}\right) \sqrt{T_{\text {wall }, i}}-n_{i} \sqrt{T_{\text {vap }, i}}\right)=0 .
\end{aligned}
$$

В левой части уравнения (4) в первых скобках положительный член - это поток в $i$-ю камеру из предыдущей $i$-1-ой камеры, отрицательный член - поток, вытекающий через щель из $i$-й камеры в $i+1$-ую (или в основной объем плазмы), во-вторых скобках положительный член - это поток лития, испаряющийся со стенок камеры, отрицательный - ленгмюровский поток литиевого пара на стенки камеры. Здесь $A_{n o z, i}-$ площадь щели между камерами $i-1$ и $i, A_{\text {wall }, i}$ - площадь поверхности стенок в камере $i$.
Следующие уравнения для баланса тепла, которое приносится и уносится парами лития в камеру с индексом , $i^{\text {““ }}(5)$, дополняют систему уравнений баланса частиц (4):

$$
\begin{aligned}
& \xi_{\mathrm{Li}} \sqrt{\frac{k^{3}}{m_{\mathrm{Li}}}} \frac{5}{2}\left(A_{n o z, i-1} n_{i-1} T_{\text {vap }, i-1}^{3 / 2}-A_{n o z, i} n_{i} T_{\text {vap }, i}^{3 / 2}\right) \\
& +\sqrt{\frac{k^{3}}{2 \pi m_{\mathrm{Li}}}} \frac{5}{2} A_{\text {wall }, i}\left(n_{e q}\left(T_{\text {wall }, i}\right) T_{\text {wall }, i}^{3 / 2}-n_{i} T_{\text {vap }, i}^{3 / 2}\right)=0 .
\end{aligned}
$$

Каждое из слагаемых для потока энтальпии имеет смысл, аналогичный потоку частиц в уравнениях баланса частиц (4).

Представленная в работе [5] модель для $N$-камерной конструкции $(N=5)$ была нами дополнена до системы из $2 N$ уравнений баланса частиц и энтальпий в камерах, которая затем решалась относительно $n_{i}$ и $T_{v a p, i}$. Помимо учета взаимодействия плазмы с литиевым паром в камерах, было учтено переизлучение мощности плазмы SOL литиевым паром.

Учет плазменного взаимодействия с поверхностью камеры 1 приводит к ее нагреву и испарению литиевых атомов. Третий член уравнения (6) описывает скорость их поступления. Она рассчитывалась из баланса мощности, поступающей из плазмы $P_{\mathrm{SOL}}$ за вычетом мощности излучения в камерах, отнесенной к энергии сублимации лития $\varepsilon_{a b l}$. Коэффициент нейтрального экранирования $\delta_{a b l}$ учитывает уменьшение мощности за счет так называемого экранирования падающей мощности испаренным веществом. Согласно работе [5], в камеру 1 попадают также литиевые частицы из камер $j=2-N$. Это происходит в результате ионизации атома лития электронами SOL-плазмы и формирования за счет трения с ионами плазмы его направленного движения в камеру 1. Предполагается, что рекомбинация ионов лития в камеры 1 увеличивает концентрацию паров лития в ней. Это описывается последним членом в уравнении (6). Соответствующее уменьшение концентрации паров лития в камерах $j=2-N$ представлено последними членами в уравнениях (7).

Учет плазмы в балансе энтальпий приводит к следующим членам в уравнениях (8), (9). В соответствии с моделью работы [5] потоки лития, ионизованные в камерах $j=2-N$, уносят из них вместе с потоками ионизованного лития часть мощности этих боксов, что описывается последним членом в уравнениях (9). Сумма этих энергий добавляется в баланс энтальпии камеры 1 , что представлено третьим членом в уравнении (8). Мощность SOL плазмы $P_{\mathrm{SOL}}$ идет на нагрев литиевого пара в камере 1. Во взаимодействии паров лития со струей плазмы процессы перезарядки $\mathrm{Li}^{0}$ на $\mathrm{Li}^{+}$и упругие столкновения атомов лития с ионами плазмы определяют передачу энергии от плазмы литиевому пару. Вслед за работой Голдстона мы предполагаем, что они доминируют в дальней от основной плазмы камере 1 
дивертора, поскольку в ней самая большая плотность и температура паров лития, а значит и потоки атомов лития на плазму. Поэтому мощность $P_{\mathrm{SOL}}$ присутствует в уравнении (8) для камеры 1 и отсутствует в уравнении (9) для остальных камер.

Вопрос оценки оптической прозрачности не входил в задачи настоящей работы, но может быть важным для расчета как нагрева пара, так и нагрузки на стенку камеры. При дальнейшем развитии модели эти оценки будут сделаны и при необходимости учтены. В настоящей работе предполагается оптическая прозрачность литиевого пара для излучения во всех камерах. То есть мощность SOL передается стенке камеры. Роль излучения в таком случае сводится к снижению мощности SOL-плазмы, что учтено последним членом в уравнениях (8). Для остальных камер излучательные потери используются для расчета соответствующей тепловой нагрузки на стенку камеры, но не учтены в уравнениях (9) энергобаланса лития в них. Цена излучательных потерь $E_{l o s s}$ варьировалась в пределах от 5 до $30 \mathrm{eV}$ на один атом лития в соответствии с расчетами столкновительноизлучательной модели работы [16].

Температуры стенок камер, являющиеся инженерным параметром, при моделировании выбирались так, чтобы мощность излучательных потерь во всех камерах дивертора компенсировала приходящую в дивертор (в камеру 1) мощность $P_{\mathrm{SOL}}$. Очевидно, что при этом в силу различия плотностей литиевых паров основной вклад вносит камера 1 (см. в табл. 1,2 значения мощностей излучательных потерь в камерах). С другой стороны, перепад температур стенок должен был обеспечить перепад давлений литиевых паров для работы в режиме дифференциальной откачки.

$$
\begin{aligned}
& \xi_{\mathrm{Li}} \sqrt{\frac{k}{m_{\mathrm{Li}}}}\left(-A_{n o z, i} n_{i} \sqrt{T_{\text {vap }, i}}\right)+\sqrt{\frac{k}{2 \pi m_{\mathrm{Li}}}} A_{\text {wall }, i} \\
& \times\left(n_{e q}\left(T_{\text {wall }, i}\right) \sqrt{T_{\text {wall }, i}}-n_{i} \sqrt{T_{\text {vap }, i}}\right) \\
& +\frac{P_{\mathrm{SOL}}-E_{\text {loss }} \sqrt{\frac{k}{2 \pi m_{\mathrm{Li}}}}\left(\sum_{j=1}^{N} A_{p l, j} n_{j} \sqrt{T_{v a p, j}}\right)}{\varepsilon_{a b l}} \delta_{a b l} \\
& +\sqrt{\frac{k}{2 \pi m_{\mathrm{Li}}}}\left(\sum_{j=2}^{N} A_{p l, j} n_{j} \sqrt{T_{\text {vap }, j}}\right)=0 \\
& \text { для } i=1 \quad \text { (камера } 1), \\
& \xi_{\mathrm{Li}} \sqrt{\frac{k}{m_{\mathrm{Li}}}}\left(A_{n o z, i-1} n_{i-1} \sqrt{T_{\text {vap }, i-1}}-A_{n o z, i} n_{i} \sqrt{T_{\text {vap }, i}}\right) \\
& +\sqrt{\frac{k}{2 \pi m_{\mathrm{Li}}}} A_{\text {wall }, i}\left(n_{e q}\left(T_{\text {wall }, i}\right) \sqrt{T_{\text {wall }, i}}-n_{i} \sqrt{T_{\text {vap }, i}}\right) \\
& -\sqrt{\frac{k}{2 \pi m_{\mathrm{Li}}}} A_{p l, i} n_{i} \sqrt{T_{\text {vap }, i}}=0 \quad \text { для } \quad i=2-N,
\end{aligned}
$$

$$
\begin{aligned}
& \xi_{\mathrm{Li}} \sqrt{\frac{k^{3}}{m_{\mathrm{Li}}}} \frac{5}{2}\left(-A_{\text {noz }, i} n_{i} T_{\text {vap }, i}^{3 / 2}\right)+\sqrt{\frac{k^{3}}{2 \pi m_{\mathrm{Li}}}} \frac{5}{2} A_{\text {wall }, i} \\
& \times\left(n_{\text {eq }}\left(T_{\text {wall }, i}\right) T_{\text {wall }, i}^{3 / 2}-n_{i} T_{\text {vap }, i}^{3 / 2}\right)+\sqrt{\frac{k^{3}}{2 \pi m_{\mathrm{Li}}}} \\
& \times \frac{5}{2} \sum_{j=2}^{N} A_{p l, j} n_{j} T_{\text {vap }, j}^{3 / 2}+P_{\mathrm{SOL}}-E_{\text {los }} \sqrt{\frac{k}{2 \pi m_{\mathrm{Li}}}} \sum_{j=1}^{N} A_{p l, j} n_{j} \\
& \times \sqrt{T_{\text {vap }, j}}=0 \quad \text { для } \quad i=1(\text { камера } 1), \\
& \xi_{\mathrm{Li}} \sqrt{\frac{k^{3}}{m_{\mathrm{Li}}}} \frac{5}{2}\left(A_{n o z, i-1} n_{i-1} T_{\text {vap }, i-1}^{3 / 2}-A_{n o z, i} n_{i} T_{\text {vap }, i}^{3 / 2}\right) \\
& +\sqrt{\frac{k^{3}}{2 \pi m_{\mathrm{Li}}}} \frac{5}{2} A_{\text {wall }, i}\left(n_{\text {eq }}\left(T_{\text {wall }, i}\right) T_{\text {wall }, i}^{3 / 2}-n_{i} T_{\text {vap }, i}^{3 / 2}\right) \\
& \quad-\sqrt{\frac{k^{3}}{2 \pi m_{\mathrm{Li}}}} \frac{5}{2} A_{p l, i} n_{i} T_{\text {vap }, i}^{3 / 2}=0 \quad \text { для } i=2-N .
\end{aligned}
$$

Здесь $A_{p l, i}=2 \times L_{i} \times 2 \pi R_{i}-$ площадь поверхности плазмы в камере $i . R_{i}$ - это усредненный большой радиус сепаратрисы в $i$-й камере (рис. 1). Фактор 2 показывает, что слой плазмы, входящий в диверторные камеры, имеет две боковые поверхности, взаимодействующие с парами лития.

Система из $N=5$ камер не подходит для ДЕМО-ТИН из-за меньших размеров установки по сравнению с разрабатываемым международным демонстрационным термоядерным реактором DEMO. Поэтому были рассчитаны параметры дивертора с испаряющими стенками с числом камер $N=2-4$.

Решения системы уравнений (6)-(9) относительно температуры и плотности паров в каждой из $N$ камер для различных значений $E_{l o s s}$ использовались для оценки длины свободного пробега атома лития

$$
\lambda_{m f p, i}=\frac{1}{\sqrt{2} d_{\mathrm{Li}}^{2} n_{i}} .
$$

Здесь, $d_{\mathrm{Li}} \cong 3 \cdot 10^{-10} \mathrm{~m}$ диаметр атома лития [15]. Результаты расчета показаны на рис. 2. Они сопоставлялись с длиной камеры вдоль сепаратрисы, представленной горизонтальной штриховой линией. Видно, что в случае двухкамерной системы обе камеры работают в газодинамическом режиме, в то время как в 3-х и 4-х-камерных конструкциях в ближайших к плазме объемах может реализоваться режим свободномолекулярного разлета. В этом случае выход лития из них ограничивался только геометрическими параметрами камер, а именно соотношением их длины и ширины выходной щели. Входящий в камеру со свободномолекулярным разлетом поток лития полагался распределенным по косинусу. В таком случае выходящий из дивертора поток можно получить, оставив из входящего только угловой диапазон, соответствующий геометрии камеры. 
Таблица 1. Основные параметры литиевого дивертора на внешней ноге в подходе с испаряющими стенками

\begin{tabular}{|c|c|c|c|}
\hline \multirow{2}{*}{ Параметры } & \multicolumn{3}{|c|}{ Количество камер } \\
\hline & 4 & 3 & 2 \\
\hline Размер камеры вдоль сепаратрисы, сm & 4 & 7.5 & 12 \\
\hline Температура стенок, начиная с камеры $1, \mathrm{~K}$ & $\begin{array}{c}1021,793 \\
723,573\end{array}$ & $984,773,473$ & 960,573 \\
\hline Температура паров Li, начиная с камеры $1, \mathrm{~K}$ & $\begin{array}{l}1052,1045 \\
1042,1041\end{array}$ & $\begin{array}{l}1006,995 \\
995\end{array}$ & 992,992 \\
\hline Давление паров Li, начиная с камеры 1, Pa & $\begin{array}{c}103,21.2 \\
4.8,1.1\end{array}$ & $59.9,7.1,0.9$ & $38.8,7.3$ \\
\hline $\begin{array}{l}\text { Мощность излучательных потерь, начиная } \\
\text { с камеры 1, MW }\end{array}$ & $\begin{array}{l}7.94,1.62 \\
0.36,0.08\end{array}$ & $\begin{array}{l}8.83,1.04 \\
0.13\end{array}$ & $9.21,0.79$ \\
\hline Максимальная тепловая нагрузка, MW/m² & 2.32 & 1.07 & 0.79 \\
\hline Поток частиц лития в SOL, mg/s & 51.7 & 41.1 & 2120 \\
\hline Поток частиц лития через сепаратрису, mg/s & 0.22 & 0.15 & 7.8 \\
\hline$P_{\mathrm{SOL}}$ в дивертор, MW & 10 & 10 & 10 \\
\hline
\end{tabular}

Таблица 2. Основные параметры литиевого дивертора на внутренней ноге в подходе с испаряющими стенками

\begin{tabular}{|c|c|c|}
\hline \multirow{2}{*}{ Параметры } & \multicolumn{2}{|c|}{ Количество камер } \\
\hline & 3 & 2 \\
\hline Размер камеры вдоль сепаратрисы, сm & 7 & 12.5 \\
\hline Температура стенок, начиная с камеры $1, \mathrm{~K}$ & $943,823,573$ & 912,573 \\
\hline Температура паров $\mathrm{Li}$, начиная с камеры $1, \mathrm{~K}$ & $957,924,924$ & 1045,1045 \\
\hline Давление паров Li, начиная с камеры $1, \mathrm{~Pa}$ & $26.5,4.8,0.7$ & $14.8,1.5$ \\
\hline $\begin{array}{l}\text { Мощность излучательных потерь, начиная } \\
\text { с камеры } 1, \text { MW }\end{array}$ & $2.39,0.53,0.08$ & $2.71,0.29$ \\
\hline Максимальная тепловая нагрузка, MW/m² & 0.62 & 0.40 \\
\hline Поток частиц лития в SOL, mg/s & 30 & 760 \\
\hline Поток частиц лития через сепаратрису, mg/s & 0.08 & 3.2 \\
\hline$P_{\mathrm{SOL}}$ в дивертор, MW & 3 & 3 \\
\hline
\end{tabular}

Необходимые для сопоставления конструкций дивертора с разным количеством камер температуры стенок камер и паров лития (перечислены через запятую, начиная с камеры 1), и основные параметры систем приведены в табл. 1 (внешняя нога дивертора) и в табл. 2 (внутренняя нога). Представленные в указанных таблицах данные получены при учете излучательных потерь на атом лития $E_{\text {loss }}=10 \mathrm{eV}$, как в работе [16]. В трехкамерном варианте, эскиз которого представлен на рис. 1, в расчете были использованы следующие площади: $A_{n o z}$ составляет $0.97,0.95,0.92 \mathrm{~m}^{2}$ для первой, второй и третьей камер соответственно; $A_{p l}$ $2.91,2.86,2.77 \mathrm{~m}^{2}$ соответственно; $A_{\text {wall }}-7.18,10.9$,
$9.05 \mathrm{~m}^{2}$ соответственно для первой, второй и третьей камер.

Для внешней ноги дивертора, параметры которого показаны в табл. 1, в случае двухкамерной системы максимальная тепловая нагрузка на стенки камер составит всего $0.8 \mathrm{MW} / \mathrm{m}^{2}$, в трехкамерной $-1.1 \mathrm{MW} / \mathrm{m}^{2}$, в четырехкамерной - $2.3 \mathrm{MW} / \mathrm{m}^{2}$. Эти значения не превышают инженерного предела $10 \mathrm{MW} / \mathrm{m}^{2}$. При увеличении числа камер за счет сохранения полной длины дивертора вдоль сепаратрисы около $40 \mathrm{~cm}$ площадь стенок камеры уменьшается, что объясняет рост тепловой нагрузки с увеличением числа камер. Эта же причина приводит к тому, что выходные потоки из трехкамерной 


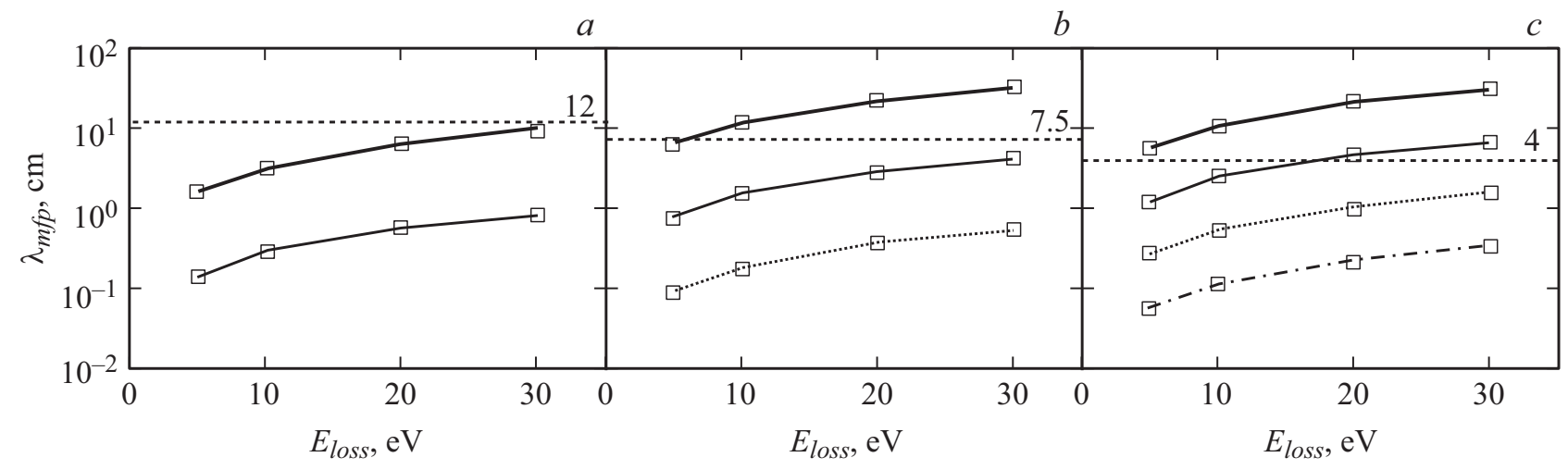

Рис. 2. Длина свободного пробега $\lambda_{m f p}$ в зависимости от излучательных потерь $E_{\text {loss }}$ в камерах для внешней ноги дивертора при числе камер $N=2(a), 3(b), 4(c)$. Значения $\lambda_{m f p}$ для ближайшей к плазме камере показаны жирной сплошной линией, далее по мере удаления от плазмы - тонкой сплошной, пунктирной и штрихпунктирной. Длина камеры вдоль сепаратрисы в сантиметрах показана горизонтальной штриховой линией.

системы оказываются меньше, чем из четырехкамерной. Действительно, уменьшение длины последней камеры, работающей в обоих случаях в свободномолекулярном режиме, приводит при той же ширине щели к увеличению диапазона углов пролета частиц напрямую сквозь камеру.

Часть покинувших дивертор атомов лития, попадая в SOL шириной $\Delta_{\mathrm{SOL}}$, испытывает ионизацию и возвращается обратно в дивертор вместе с потоком дейтериевой плазмы из SOL. Характерная длина ионизации $l_{\text {ion }}$ зависит как от скорости вылетающих частиц $V_{\mathrm{Li}}$, так и от температуры и плотности плазмы SOL:

$$
l_{\text {ion }}=\frac{V_{\mathrm{Li}}}{\left\langle\sigma v_{\text {ion }}\left(T_{\mathrm{SOL}}\right)\right\rangle n_{\mathrm{SOL}}},
$$

где скорость ионизации лития рассчитывалась с помощью аппроксимации из работы [25].

Оценка длины ионизации имеет кинетический характер, поэтому применимость ее к выходящему из дивертора потоку лития требует подтверждения. Этот поток после выхода из последней камеры претерпевает расширение. Предположим, что он истекает из выходной щели со скоростью звука $V_{s}$, соответствующей температуре паров в камере, а расширяется с тепловой скоростью $V_{T}$. Тогда можно оценить, насколько он расширится, преодолев расстояние $L=30 \mathrm{~cm}$ от дивертора до сепаратрисы: $\kappa=\frac{L \cdot\left(V_{T} / V_{s}\right)+H / 2}{H / 2}=17.1$, где $H-$ ширина щели на выходе из последней камеры дивертора. Во столько же раз упадет и давление паров лития. Тогда длина свободного пробега его паров составит около $10 \mathrm{~cm}$, что значительно больше ширины SOL. Это позволяет применить кинетическую оценку проникновения атомов лития через SOL.

Таким образом, по аналогии с [15] можно получить коэффициент экранирования за счет ионизации в SOL:

$$
\delta_{\mathrm{SOL}}=\exp \left(-\Delta_{\mathrm{SOL}} / l_{\text {ion }}\right)
$$

Тогда поток лития в основную плазму $\Gamma_{\mathrm{Li}}$ уменьшится в $\delta_{\mathrm{SOL}}$ раз по сравнению с потоком, выходящим из дивертора в SOL $\Gamma_{\mathrm{Li}, \mathrm{SOL}}: \Gamma_{\mathrm{Li}=\Gamma_{\mathrm{Li}, \mathrm{SOL}}} \cdot \delta_{\mathrm{SOL}}$.

Очевидно, что ширина SOL $\Delta_{\mathrm{SOL}}$ играет ключевую роль в данных оценках. Исходя из современного скейлинга работы [4], для параметров ДЕМО-ТИН она составит $0.15 \mathrm{~cm}$, а с учетом рассчитанного по коду DINA магнитного расширения [20] $\Delta_{\mathrm{SOL}}$ будет равно $0.3 \mathrm{~cm}$, что является оценкой снизу, поскольку она не учитывает уширение SOL за счет поперечного переноса тепла и частиц. Подставив в формулу (11) температуру плазмы и еe плотность в SOL в соответствии с упомянутыми выше оценками $T_{p l}=150 \mathrm{eV}$, $n_{p l}=4 \cdot 10^{13} \mathrm{~cm}^{-3}$, получим $l_{\text {ion }} \approx 0.054 \mathrm{~cm}$. В этом случае фактор ионизационного экранирования, согласно (12), составляет $\delta_{\mathrm{SOL}}=3.7 \cdot 10^{-3}$. Это показывает, что проникновение лития в центральные области плазмы за счет ионизации в плазме SOL может уменьшиться на несколько порядков.

В результате для внешней нижней ноги дивертора в двухкамерной схеме поток лития в центральную область плазмы составит $7.8 \mathrm{mg} / \mathrm{s}$, в трехкамерной $-0.15 \mathrm{mg} / \mathrm{s}$, в четырехкамерной $-0.22 \mathrm{mg} / \mathrm{s}$ (табл. 1). Для дивертора на внутренней нижней ноге поток лития в основную плазму составит $3.2 \mathrm{mg} / \mathrm{s}$ для двухкамерной системы и $0.08 \mathrm{mg} / \mathrm{s}$ для трехкамерной (табл. 2). В результате, если просуммировать потоки лития от всех четырех ног дивертора, то в оптимальном варианте - трехкамерные системы как на внутренней, так и на внешней ноге суммарный поток лития достигнет $0.5 \mathrm{mg} / \mathrm{s}$, что не превышает оцененный выше предел $4.5 \mathrm{mg} / \mathrm{s}$.

Необходимо отметить, что оценка с помощью уравнения (12) экранирующих свойств SOL крайне упрощенная. Эти свойства зависят от распределения концентрации и температуры поперек магнитного поля и вдоль тороидального направления SOL. Например, в точке стагнации потоки частиц вдоль магнитного поля равны нулю и в этой области уход лития на диверторные 
пластины маловероятен, скорее за счет перезарядки он пройдет через сепаратрису в основную плазму. Кроме того, параметры плазмы в проекте ДЕМО-ТИН, определяющие значение $\delta_{\mathrm{SOL}}$, пока известны с недостаточной точностью. Необходимо подчеркнуть, что при изменении ширины SOL на $1 \mathrm{~mm}(33 \%)$ поток лития в плазму изменится на порядок (см. уравнение $(12)$ ). Ширина $\delta_{\mathrm{SOL}}$ зависит от геометрии установки и параметров выбранной магнитной системы. Более того, толщина SOL зависит от полоидального угла. Так что для уточнения экранирующих свойств SOL в ДЕМО-ТИН необходимо двумерное моделирование переноса лития с помощью кода типа SOLPS для описанной конструкции дивертора. Значения плотности и температуры в SOL также играют заметную роль в расчете.

Из табл. 1 видно, что потоки лития из камер дивертора в плазму SOL невелики по сравнению с оцененным выше предельным потоком $0.21 \mathrm{~g} / \mathrm{s}$. Так, в наилучшем случае (3-х-камерный дивертор как на внешней, так и на внутренней ноге) суммарный поток составляет $0.15 \mathrm{~g} / \mathrm{s}$, что не должно привести к заметному выхолаживанию SOL.

Важной проблемой данной системы является необходимость поддерживать постоянно высокие температуры стенок камер $(\sim 1000 \mathrm{~K})$, что технически реализовать сложнее, чем вариант с конденсирующей стенкой с более низкими температурами. Таким образом, подход с испаряющими стенками для текущего дизайна дивертора ДЕМО-ТИН выглядит проблематичным с инженерной точки зрения.

\section{3. Оценки для дивертора ДЕМО-ТИН по подходу с конденсирующими стенками}

Как уже упоминалось выше, в исходной работе Нагаямы [15] предполагались условия свободномолекулярного разлета испаряемого лития в двухкамерной системе с холодными (около $200^{\circ} \mathrm{C}$, т. е. чуть выше температуры плавления лития) стенками. Приходящий из SOL вдоль сепаратрисы тепловой поток приводит к активному испарению жидкого лития, находящегося в бассейне в дальней от плазмы камере дивертора (камеpa 1 на рис. 1). Максимальную величину испаряемого потока можно ценить как $10 \mathrm{MW} / \varepsilon_{a b l} \cong 4.1 \cdot 10^{25}$ частиц в секунду $(\approx 0.48 \mathrm{~kg} / \mathrm{s})$ для внешней ноги дивертора и $1.2 \cdot 10^{25}$ частиц в секунду $(\approx 0.14 \mathrm{~kg} / \mathrm{s})$ для внутренней ноги при поступающей сюда мощности $3 \mathrm{MW}$. Здесь $\varepsilon_{a b l}=1.5 \mathrm{eV}$ - энергия сублимации атома лития. Видно, что эти значительные потоки должны быть снижены как минимум на 3 порядка величины.

Облако частиц, возникающее над литиевым бассейном за счет испарения, будет поглощать часть падающей мощности и уменьшать испарение. Расчет процесса требует двумерного моделирования, однако предварительные оценки можно выполнить, используя модель нейтрального экранирования [26]. Положив размер испаряемой области $\sim 1 \mathrm{~cm}$ и угол наклона силовой линии в области точки удара (strike point) 1/10 rad, получаем значение коэффициента нейтрального экранирования $\delta_{a b l}=0.04-0.1$. Таким образом, концентрация паров лития в камерах дивертора может быть снижена более чем на порядок по сравнению с оценками максимальных скоростей испарения.

Для описанной в предыдущем параграфе конструкции дивертора, плотности паров лития были оценены с помощью уравнений (6) и (7). Это позволило рассчитать длины свободного пробега атомов лития в каждой из камер диверторов ДЕМО-ТИН. Для дивертора на внешней ноге (входящая мощность $10 \mathrm{MW}$ ) при $\delta_{a b l}=0.04$ в камерах 1, 2 и 3 длина свободного пробега составляет 1, 12 и $93 \mathrm{~cm}$ соответственно. Таким образом, в камере 1 реализуется газодинамический режим (длина свободного пробега много меньше характерного размера камеры $7.5 \mathrm{~cm})$, в камерах 2 и 3 - режим свободномолекулярного разлета. Подчеркнем, что это расходится с предположениями работы [15], где свободномолекулярный разлет ожидался даже в камере с бассейном-испарителем. При $\delta_{a b l}=0.1$ длины свободного пробега соответственно в камерах 1, 2 и 3 будут 0.5, 5 и $37 \mathrm{~cm}$, т.е. теперь получается две газодинамические камеры (из которых вторая за счет низкой температуры стенок работает как дифференциальная откачка) и одна свободномолекулярная камера. Для дивертора на внутренней ноге поступающей мощности $3 \mathrm{MW}$ также будет достаточно для реализации газодинамического режима только в камере 1 во всем оцененном диапазоне $\delta_{a b l}$. Температура паров лития на выходе из камер с газодинамическим режимом полагалась близкой к температуре стенок камеры.

Для камер, в которых выполнялся газодинамический режим, были получены значения выходящего потока паров лития $\Gamma_{n, 1}$ согласно уравнению (1). Затем, используя геометрические размеры конструкции дивертора ДЕМО-ТИН (апертуры выходных отверстий и длину вдоль сепаратрисы тех камер, где реализуется свободномолекулярный разлет), можно определить, насколько этот поток ограничивается в камерах дифференциальной откачки (рис. 1). Для дивертора на внешней ноге получаем коэффициент геометрического экранирования $\delta_{\text {geom }}=0.10$ при двух свободномолекулярных камерах и 0.18 при одной (табл. 3), а для дивертора на внутренней ноге $\delta_{\text {geom }}=0.13$ при 2 свободномолекулярных камерах (табл. 4).

Необходимо отметить, что использование искривленной сепаратрисы, схематично показанной на рис. 3, выглядит еще более привлекательно, так как при воздействии мощных потоков в дивертор возникающие при прямом разбрызгивании капли расплавленного металла не смогут попасть назад в плазму. Однако возможность технической реализации такой магнитной конфигурации в ДЕМО-ТИН требует дополнительных расчетов с помощью кода DINA [27]. 
Таблица 3. Основные параметры трехкамерного литиевого дивертора на внешней ноге в подходе с конденсирующими стенками

\begin{tabular}{|c|c|c|}
\hline Параметры & $\begin{array}{c}\text { Коэффициент } \\
\text { нейтрального } \\
\text { экранирования } \delta_{a b l}=0.1\end{array}$ & $\begin{array}{c}\text { Коэффициент } \\
\text { нейтрального } \\
\text { экранирования } \delta_{a b l}=0.04\end{array}$ \\
\hline $\begin{array}{l}\text { Коэффициент } \\
\text { геометрического } \\
\text { экранирования } \delta_{\text {geom }}\end{array}$ & 0.18 & 0.10 \\
\hline $\begin{array}{l}\text { Поток частиц лития из } \\
\text { камеры с бассейном-испарителем, } \\
\mathrm{g} / \mathrm{s}\end{array}$ & 0.33 & 1.2 \\
\hline $\begin{array}{l}\text { Коэффициент } \\
\text { ионизационного } \\
\text { экранирования } \delta_{\mathrm{SOL}}\end{array}$ & $3.7 \cdot 10^{-4}$ & $3.7 \cdot 10^{-4}$ \\
\hline Поток частиц лития в SOL, g/s & 0.06 & 0.12 \\
\hline $\begin{array}{l}\text { Поток частиц лития через } \\
\text { сепаратрису, mg/s }\end{array}$ & 0.022 & 0.045 \\
\hline$P_{\mathrm{SOL}}$ в дивертор, MW & 10 & 10 \\
\hline
\end{tabular}

Таблица 4. Основные параметры трехкамерного литиевого дивертора на внутренней ноге в подходе с конденсирующими стенками

\begin{tabular}{|c|c|c|}
\hline Параметры & $\begin{array}{c}\text { Коэффициент } \\
\text { нейтрального } \\
\text { экранирования } \delta_{a b l}=0.1\end{array}$ & $\begin{array}{c}\text { Коэффициент } \\
\text { нейтрального } \\
\text { экранирования } \delta_{a b l}=0.04\end{array}$ \\
\hline $\begin{array}{l}\text { Коэффициент } \\
\text { геометрического } \\
\text { экранирования } \delta_{\text {geom }}\end{array}$ & 0.13 & 0.13 \\
\hline $\begin{array}{l}\text { Поток частиц лития из } \\
\text { камеры с бассейном-испарителем, } \\
\mathrm{g} / \mathrm{s}\end{array}$ & 0.92 & 0.37 \\
\hline $\begin{array}{l}\text { Коэффициент } \\
\text { ионизационного } \\
\text { экранирования } \delta_{\mathrm{SOL}}\end{array}$ & $3.7 \cdot 10^{-4}$ & $3.7 \cdot 10^{-4}$ \\
\hline Поток частиц лития в SOL, g/s & 0.12 & 0.048 \\
\hline $\begin{array}{l}\text { Поток частиц лития через } \\
\text { сепаратрису, mg/s }\end{array}$ & 0.044 & 0.018 \\
\hline$P_{\mathrm{SOL}}$ в дивертор, MW & 3 & 3 \\
\hline
\end{tabular}

В результате в диверторной системе с конденсирующими стенками значение продольного потока тепла на входе в дивертор $\sim 1 \mathrm{GW} / \mathrm{m}^{2}$ снижается за счет переизлучения на литиевых парах в камерах дивертора. Значения потока тепла на внутренних поверхностях секций (камер) литиевого испарителя при этом не превышают $0.5 \mathrm{MW} / \mathrm{m}^{2}$. Для оценки выхода лития в SOL и в основную плазму разумно использовать наименее выгодную для нас комбинацию параметров $\delta_{g e o m}$ и $\delta_{a b l}$ из представленных в табл. 3 и 4 значений. Поток в SOL рассчитывался как $\Gamma_{\mathrm{Li}, \mathrm{SOL}}=\Gamma_{n, 1} \cdot \delta_{\text {geom }}$, а поток в основную плазму как $\Gamma_{\mathrm{Li}}=\Gamma_{\mathrm{Li}, \mathrm{SOL}} \cdot \delta_{\mathrm{SOL}}$. Ha внутренней ноге дивертора наименее удачной комбина- цией являются условия, дающие значения потока лития в $\mathrm{SOL} \cong 0.12 \mathrm{~g} / \mathrm{s}$ при $\delta_{a b l}=0.1$. Соответственно для внешней ноги значения потока $\cong 0.06 \mathrm{~g} / \mathrm{s}$ при $\delta_{a b l}=0.1$. В итого суммарный поток лития из всех 4 диверторов даст значений $\cong 0.36 \mathrm{~g} / \mathrm{s}$ на входе в SOL. Это значение несколько выше предела в $0.21 \mathrm{~g} / \mathrm{s}$, оцененного в разд. 1 настоящей работы, и может привести к выхолаживанию SOL. C другой стороны, радиационное охлаждение периферии за счет внесения туда лития может снизить тепловую нагрузку на обращенные к плазме элементы установки. Приведенные выше оценки очень упрощенные, и дальнейшее их уточнение требует дополнительных расчетов с использованием кодов, в 


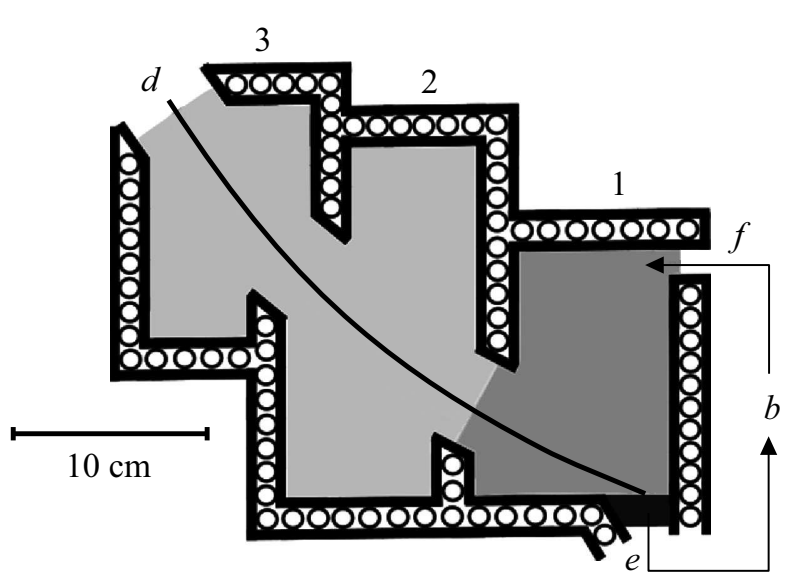

Рис. 3. Принцип устройства литиевого дивертора в подходе с конденсирующими стенками с изогнутой сепаратрисой. Цифрами показаны номера камер: 1 - камера с бассейномиспарителем, 2, 3 - камеры дифференциальной откачки. $b$ - система очистки и перекачки лития, $d$ - сепаратриса, $e$ и $f$ - выход лития в систему очистки и перекачки, и вход чистого лития в дивертор соответственно.

которых движение атомов рассчитывается в столкновительном, бесстолкновительном и переходном между ними режимах.

Потоки в основную плазму (с учетом ионизационного экранирования, описанного в предыдущем параграфе) наибольшие при тех же условиях, что и потоки в SOL. Это соответствует значениям $0.044 \mathrm{mg} / \mathrm{s}$ для внутренней ноги и $0.0221 \mathrm{mg} / \mathrm{s}$ для внешней. Суммарный для верхнего и нижнего диверторов поток составляет $0.132 \mathrm{mg} / \mathrm{s}$, что ниже предельного значения $4.5 \mathrm{mg} / \mathrm{s}$. Таким образом, подход с конденсирующими стенками удовлетворяет трем из вышеперечисленных четырех критериев применимости на ДЕМО-ТИН. Критерию по потоку лития в основную плазму он также удовлетворяет полностью, а критерий по потоку лития в SOL превышает не сильно. Необходимо подчеркнуть, что более низкие температуры камер порядка $200^{\circ} \mathrm{C}$ делают данный подход привлекательнее с инженерной точки зрения.

При использовании в ДЕМО-ТИН двухнулевой магнитной конфигурации требуется обеспечить достаточное количество испаряемого лития в камере 1. Для этого для верхних диверторных пластин предполагается вместо бассейна-испарителя использовать поток расплавленного лития, протекающий по поддерживаемой при заданной температуре направляющей пластине из нержавеющей стали, наподобие конструкции, описанной в работе [28]. Также возможно рассмотреть конструкцию на основе КПС [13]. Следует отметить, что поверхностный поток жидкого лития может быть неустойчив к срывам плазменного разряда, и проблема восстановления работоспособности такой конструкции требует дальнейших исследований. Капиллярно-пористые структуры представляются предпочтительнее с этой точки зрения.

\section{4. Обсуждение}

Исходя из сформулированных выше критериев, на данный момент наиболее приемлемым для текущего дизайна установки ДЕМО-ТИН является подход секционированного литиевого испарителя с конденсирующими стенками. Для него поток лития из диверторной системы в основную плазму ожидается примерно на порядок меньше оцененных предельных значений. Кроме того, организация циркуляции лития в диверторном объеме в подходе с конденсирующими стенками представляется более простой с инженерной точки зрения, чем в подходе с испаряющими стенками. Отметим, что организация замкнутого цикла с циркуляцией контактирующего с плазмой слоя является важным преимуществом жидкометаллических поверхностей первой стенки и дивертора по сравнению с твердотельными тайлами, например, из бериллия, как отмечалось в работе [21]. В таком случае нет проблем с потоками плазмы, которые могут вызывать эрозию материала тайла, а также с его загрязнением при длительной квазистационарной работе будущих термоядерных установок.

Необходимо отметить, что выше рассматривались варианты дивертора в условиях стационарные тепловых нагрузок. К настоящему моменту неясно, в каком режиме - c ELM (Edge-Localized Mode) или без них будет работать ДЕМО-ТИН. Если в рабочем режиме, с наибольшим временем удержания энергии, будут присутствовать ELM I типа, это будет сопровождаться дополнительными нестационарными (с длительностью до $\mathrm{ms}$ для ДЕМО-ТИН) потоками тепла и плазмы в дивертор и на первую стенку, амплитуды которых в несколько раз могут превышать соответствующие значения для стационарных условий. Необходимы расчеты и разработки мер для работы диверторной системы в таких условиях или мер для смягчения последствий ELM I типа, которые являются опасными, как показано в экспериментах с ILW первой стенкой на токамаке JET [29], для наиболее близких к условиям ДЕМО-ТИН параметрам плазмы.

Развитие изложенной модели дивертора будет включать учет процессов перезарядки, которая может играть роль не только в первой камере. Учет распределения $P_{\mathrm{SOL}}$ по камерам дивертора может потребовать изменить соотношения температур стенок камеры для оптимизации работы дивертора. Кроме того, в модели с конденсирующими стенками необходимо усовершенствовать расчет самосогласованного испарения лития в диверторе под воздействием SOL-плазмы. Такой анализ предполагается сделать в будущей улучшенной версии модели.

Более детальные двумерные расчеты вариантов диверторной системы для ДЕМО-ТИН в подходе с конденсирующими стенками предполагается сделать с помощью кода SOLPS4.3, который используется для гибридного моделирования ДЕМО-ТИН [19,20]. Для этого предполагается согласовать граничные условия для кода 
SOLPS4.3 и для нашей модели на поверхностях дивертора, обращенных к плазме.

\section{Заключение}

Проведено сравнение характеристик двух перспективных конструкций секционированных литиевых диверторов для токамака ДЕМО-ТИН. В рамках сделанных в работе предположений и выполненных оценок система с более холодными конденсирующими литий стенками видится более привлекательной, чем конструкция с горячими испаряющими стенками. Двум критериям (по тепловой нагрузке на стенки дивертора, и по возможности размещения внутри текущего дизайна дивертора ДЕМО-ТИН) удовлетворяют оба подхода. Критерию по потоку лития в основную плазму также удовлетворяют оба подхода, но подход с конденсирующими стенками находится на грани применимости по потоку лития в SOL. По инженерной простоте реализации подход с конденсирующими стенками является предпочтительным. Оценки показывают, что снижение потоков лития из дивертора через сепаратрису в основную плазму в основном определяют экранирующие свойства плазмы SOL. Результаты выполненных оценок предполагается уточнить в рамках дальнейшей работы, для чего будет выполнено двумерное моделирование параметров SOL и дивертора с литиевыми камерами в условиях ДЕМО-ТИН с помощью кодов семейства SOLPS. Планируется провести дополнительную инженерно-техническую проработку предлагаемых конструкций.

\section{Благодарности}

Настоящая работа поддержана Министерством науки и высшего образования Российской Федерации в рамках государственного задания в сфере науки по проекту № 0784-2020-0020 с использованием Федерального центра коллективного пользования „Материаловедение и диагностика в передовых технологиях“ (проект RFMEFI62119Х0021), включающего Уникальную научную установку „Сферический токамак Глобус-М“.

\section{Конфликт интересов}

Авторы заявляют, что у них нет конфликта интересов.

\section{Список литературы}

[1] A. Loarte, B. Lipschultz, A.S. Kukushkin, G.F. Matthews, P.C. Stangeby, N. Asakura, G.F. Counsell, G. Federici, A. Kallenbach, K. Krieger, A. Mahdavi, V. Philipps, D. Reiter, J. Roth, J. Strachan, D. Whyte, R. Doerner, T. Eich, W. Fundamenski, A. Herrmann, M. Fenstermacher, P. Ghendrih, M. Groth, A. Kirschner, S. Konoshima, B. LaBombard, P. Lang, A.W. Leonard, P. Monier-Garbet, R. Neu, H. Pacher, B. Pegourie, R.A. Pitts, S. Takamura, J. Terry, E. Tsitrone, ITPA Scrape-off Layer and Divertor Physics Topical Group. Nucl. Fusion, 47, S203 (2007). https://doi.org/10.1088/0029-5515/47/6/S04
[2] Б.В. Кутеев, П.Р. Гончаров, В.Ю. Сергеев, В.И. Хрипунов. Физика плазмы, 36 (4), 307 (2010). [B.V. Kuteev, P.R. Goncharov, V.Yu. Sergeev, V.I. Khripunov. Plasma Phys. Rep. 36, 281 (2010). https://doi.org/10.1134/S1063780X1004001X]

[3] B.V. Kuteev, E.A. Azizov, A.S. Bykov, A.Yu. Dnestrovsky, V.N. Dokuka, G.G. Gladush, A.A. Golikov, P.R. Goncharov, M. Gryaznevich, M.I. Gurevich, A.A. Ivanov, R.R. Khairutdinov, V.I. Khripunov, D. Kingham, A.V. Klishchenko, V.A. Kurnaev, V.E. Lukash, S.Yu. Medvedev, P.V. Savrukhin, V.Yu. Sergeev, Yu.S. Shpansky, A. Sykes, G. Voss, A.V. Zhirkin. Nucl. Fusion, 51, 073013 (2011). https://doi.org/10.1088/0029-5515/51/7/073013

[4] T. Eich, B. Sieglin, A. Scarabosio, W. Fundamenski, R.J. Goldston, A. Herrmann. J. Phys. Rev. Lett., 107, 215001 (2011). https://doi.org/10.1103/PhysRevLett.107.215001

[5] R.J. Goldston, R. Myers, J. Schwartz. Phys. Scr., 167, 014017 (2016). https://doi.org/10.1088/0031-8949/T167/1/014017

[6] A. Herrmann. Plasma Phys. Control. Fusion, 44, 883 (2002). https://doi.org/10.1088/0741-3335/44/6/318

[7] A.W. Leonard. Plasma Phys. Control. Fusion, 60, 044001 (2018). https://doi.org/10.1088/1361-6587/aaa7a9

[8] P.M. Valanju, M. Kotschenreuther, S.M. Mahajan, J. Canik. Phys. Plasmas, 16, 056110 (2009). https://doi.org/10.1063/1.3110984

[9] D.D. Ryutov. Phys. Plasmas, 14, 064502 (2007). https://doi.org/10.1063/1.2738399

[10] S.V. Mirnov. Plasma Phys. Control. Fusion, 55, 045003 (2013). https://doi.org/10.1088/0741-3335/55/4/045003

[11] G. Mazzitelli et al., 44th EPS Conf. Plasma Phys., Belfast, 26-30 June 2017, O5.132.

[12] S.I. Krasheninnikov, L.E. Zakharov, G.V. Pereverzev. Phys. Plasmas, 10, 1678 (2003). https://doi.org/10.1063/1.1558293

[13] A. Vertkov, I. Luyblinski, V. Evtikhin, G. Mazzitelli, M.L. Apicella, V. Lazarev, A. Alekseyev, S. Khomyakov. Fusion Eng. Des., 82, 1627 (2007). https://doi.org/10.1016/j.fusengdes.2007.05.009

[14] M.A. Jaworski, T. Abrams, J.P. Allain, M.G. Bell, R.E. Bell, A. Diallo, T.K. Gray, S.P. Gerhardt, R. Kaita, H.W. Kugel, B.P. LeBlanc, R. Maingi, A.G. Mc Lean, J. Menard, R. Nygren, M. Ono, M. Podesta, A.L. Roquemore, S.A. Sabbagh, F. Scotti, C.H. Skinner, V.A. Soukhanovskii, D.P. Stotler and the NSTX Team. Nucl. Fusion, 53, 083032 (2013). https://doi.org/10.1088/0029-5515/53/8/083032

[15] Y. Nagayama. Fusion Eng. Des., 84, 1380 (2009). https://doi.org/10.1016/j.fusengdes.2009.02.002

[16] R.J. Goldston, A. Hakim, G.W. Hammett, M.A. Jaworski, J. Schwartz. Nucl. Mater. Energy, 12, 1118 (2017). https://doi.org/10.1016/j.nme.2017.03.020

[17] B.V. Kuteev, Yu.S. Shpanskiy, DEMO-FNS Team, Nucl. Fusion, 57, 076039 (2017). https://doi.org/10.1088/1741-4326/aa6dcb

[18] Yu.S. Shpanskiy, DEMO-FNS Project Team, Nucl. Fusion, 59, 076014 (2019). https://doi.org/10.1088/1741-4326/ab14a8

[19] A.Y. Dnestrovskiy, A.S. Kukushkin, B.V. Kuteev, V.Y. Sergeev. Nucl. Fusion, 59, 096053 (2019). https://doi.org/10.1088/1741-4326/ab3075

[20] A.S. Kukushkin, V.Yu. Sergeev, B.V. Kuteev. J. Physics: Conf. Series, 907, 012012 (2017). https://doi.org/10.1088/1742-6596/907/1/012012 
[21] V.Yu. Sergeev, B.V. Kuteev, A.S. Bykov, A.A. Gervash, D.A. Glazunov, P.R. Goncharov, A.Yu. Dnestrovskij, R.R. Khayrutdinov, A.V. Klishchenko, V.E. Lukash, I.V. Mazul, P.A. Molchanov, V.S. Petrov, V.A. Rozhansky, Yu.S. Shpanskiy, A.B. Sivak, V.G. Skokov, A.V. Spitsyn. Nucl. Fusion, 55, 123013 (2015).

https://doi.org/10.1088/0029-5515/55/12/123013

[22] Y.A. Çengel, J.M. Cimbala. Fluid Mechanics: Fundamentals and Applications (Mc Graw-Hill, N Y., 2006)

[23] E.D. Emdee, R.J. Goldston, J.A. Schwartz, M.E. Rensink, T.D. Rognlien. Nucl. Mater. Energy, 19, p. 244 (2019). https://doi.org/10.1016/j.nme.2019.01.032

[24] W.H. Chubberly. Metals handbook (American Society for Metals, 1979), v. 2.

[25] K.L. Bell, H.B. Gilbody, J.G. Hughes, A.E. Kingston, F.J. Smith. J. Phys. Chem. Ref. Data, 12 (4), 891 (1983). https://doi.org/10.1063/1.555700

[26] В.Ю. Сергеев, О.А. Бахарева, Б.В. Кутеев, М. Тендлер. Физика плазмы, 32 (5), 398 (2006). [V.Yu. Sergeev, O.A. Bakhareva, B.V. Kuteev, M. Tendler. Plasma Phys. Rep. 32, 363 (2006). https://doi.org/10.1134/S1063780X06050023]

[27] R.R. Khayrutdinov, V.E. Lukash. J. Comput. Phys., 109, 193 (1993). https://doi.org/10.1006/jcph.1993.1211

[28] G.Z. Zuo, J.S. Hu, R. Maingi, J. Ren, Z. Sun, Q.X. Yang, Z.X. Chen, H. Xu, K. Tritz, L.E. Zakharov, C. Gentile, X.C. Meng, M. Huang, W. Xu, Y. Chen, L. Wang, N. Yan, S.T. Mao, Z.D. Yang, J.G. Li, EAST Team. Nucl. Fusion, 57, 046017 (2017). https://doi.org/10.1088/1741-4326/aa5ea0

[29] W. Fundamenski et al., EFDA-JET-CP(08)05/15 (2008). 